\title{
Role of Fine needle aspiration cytology in the diagnosis of Cervical lymphadenopathy
}

\author{
Md. Arif Hossain Bhuyan¹, Md. Abu Yusuf Fakir², ABM.Tofazzal Hossain ${ }^{3}$, AHM Zahurul Huq ${ }^{4}$, \\ Sanjeev Gupta $^{5}$
}

\begin{abstract}
:
Objective: A study was carried out to know the overall prevalence of various causes responsible for cervical lymphadenopathy of more than 3 weeks duration. FNAC has been evaluated as a diagnostic tool in our clinical set up. We aimed to present 110 cases which we treated in our center within last $3 \frac{1}{2}$ years.

Methods: A retrospective study has been carried out from June 2005 to December 2008 in the department of Otolaryngology and Head-Neck Surgery at Apollo Hospitals Dhaka. All patients presented with cervical lymphadenopathy of more than 3 weeks duration. FNAC has been done for all suspected cases. CT neck has been done in 4 cases for deep jugular nodes. 55 patients underwent biopsy as FNAC report was non-specific lymphadenitis. For three cases we have done frozen section biopsy. Preoperative workup with routine blood tests, Xray chest and tuberculin tests have been carried out for all cases.

Results: Out of 110 FNAC of cervical lymphadenopathy - 55 were non-specific lymphadenitis, 32 were tuberculosis, 12 were metastatic (with 2 occult primary), 6 were lymphomas(Immunocytochemistry proved) and 5 were abscess. All 55 non-specific lymphadenopathy cases (FNAC report) underwent lymph node biopsy. 5 cases were consistent with tuberculosis, 1 was lymphoma and rest were reactive. For 3 cases frozen section biopsy have been done. One was consistent with granulomatous disease and two cases were diagnosed as lymphoid hyperplasia. Both the lymphoid hyperplasia cases have been diagnosed as Castleman's disease after histopathology report.

Conclusion: The study concluded the fact that the non-specific infection is the most common cause of cervical lymphadenopathy followed by tuberculosis. Supplemented with routine laboratory investigations, FNAC give very important clue to the physicians among patients presented with cervical lymphadenopathy.
\end{abstract}

Key Words : Lymphadenopathy, lymphoid hyperplasia, tuberculosis, lymphoma, castleman's disease.

\section{Introduction}

Lymphadenopathy is an abnormal increase in size and/ or altered consistency of lymph nodes. It is a clinical manifestation of regional or systemic disease

1. Senior Registrar, Dept. of Otolaryngology and HeadNeck Surgery, Apollo Hospitals Dhaka

2. Registrar, Dept. of Otolaryngology and Head- Neck Surgery, Dhaka Medical College Hospital.

3. Registrar, Dept. of Otolaryngology and Head- Neck Surgery, Apollo Hospitals Dhaka

4. Consultant, Dept. of Otolaryngology and Head- Neck Surgery, Bangabandhu Sheikh Mujib Medical University, Shahbag, Dhaka.

5. Consultant, Dept. of Otolaryngology and Head- Neck Surgery, Apollo Hospitals Dhaka.

Address of correspondence : Dr. Md. Arif Hossain Bhuyan, Senior Registrar, Dept. of Otolaryngology and Head-Neck Surgery, Apollo Hospitals Dhaka. E-mail: arifpp74@yahoo.com and serves as an excellent clue to the underlying disease. Cervical lymphadenopathy is a fairly common clinical presentation. It is often a diagnostic challenge to medical professionals. The present study try to give an idea to proceed with such cases and also, to give insight to the medical professional about the diagnostic outcome and role of FNAC (Fine needle Aspiration cytology ) among patients presented with cervical lymphadenopathy of more than 3 weeks duration. Cervical lymphadenopathy can be presented as isolated or as a part of generalized lymphadenopathy. The use of fine needle aspiration cytology (FNAC) in the investigation of lymphadenopathy has become an acceptable and widely practiced minimally invasive technique, which is safe, simple, rapid and relatively pain-free. FNAC is highly cost effective and accurate as a first line investigative technique with differential diagnosis including reactive hyperplasia/inflammatory conditions, 
granulomatous disorders and malignancy, stratifying cases requiring further investigations, surgical intervention or clinical follow-up ${ }^{1}$.

\section{Patients and Methods}

A retrospective study has been carried out from June 2005 to November 2008 in department of Otolaryngology and Head-Neck Surgery at Apollo Hospitals Dhaka. All patients presented with cervical lymphadenopathy. FNAC has been done for cervical lymphadenopathy of more than 3 weeks duration. CT neck has been done in 4 cases for deep jugular nodes. 55 patients underwent biopsy as FNAC report was non-specific. For three cases we have done frozen section biopsy. Imprints have been taken from those frozen section biopsies and later on immunocytochemistry have also been done. Preoperative workup with routine blood tests, X-ray chest and tuberculin tests have carried out for all cases.

\section{Results}

Many of our patients presented with cervical lymphadenopathy from June 2005 to November 2008. FNAC has been done in cases of cervical lymphadenopathy of more than 3 weeks duration. 110 cases have been sent for FNAC. Out of 110 FNAC of cervical lymphadenopathy, 55 were non-specific lymphadenitis, 32 were tuberculosis, 12 were metastatic (with 2 occult primary), 6 were lymphomas (Immunocytochemistry proved) and 5 were abscess. All 55 non-specific lymphadenopathy cases (FNAC report) underwent lymph node biopsy. Out of them 5 cases were consistent with tuberculosis, 1 was lymphoma and rest were reactive. For 3 cases, frozen section biopsy have been done. One was consistent with granulomatous disease and two cases were diagnosed as lymphoid hyperplasia. Both the lymphoid hyperplasia cases have been diagnosed as Castleman's disease after histopathology report. There were no systemic manifestations for both the cases.

\section{Discussion}

The well-defined role of FNAC in the investigation of lymphadenopathy has previously been studied ${ }^{2}$. In the context of granulomatous disorders, the possible aetiology is wide and the use of FNAC with other ancillary tests (microbiological, immunohistochemical, radiological, biochemical and special staining techniques) is useful for obtaining a definitive diagnosis $^{3}$. In our study 32 cases $(29.09 \%)$ were diagnosed as tuberculosis on FNAC. This has been recommended as of first line screening method in suspected malignancy ${ }^{4}$.In our study 18 cases have been diagnosed as malignancy on FNAC. Out of those 12 cases (10.9\%) were metastatic and 6 cases $(5.45 \%)$ were lymphoma. The presence of granulomata in an aspirate may indicate the presence of a neoplastic process. Granulomata may be encountered in both Hodgkin's disease and nonHodgkin's lymphoma, particularly T-cell lymphoma ${ }^{4}$. Hodgkin's lymphoma is characterised by the classic Reed-Sternberg cells in a background of sarcoid-like granulomata, reactive lymphoid cells and occasional eosinophils ${ }^{5}$. Occasionally, lymph nodes containing metastatic carcinoma may also show features of granulomata. Previous reports have been described in metastatic nasopharyngeal carcinoma, seminoma and malignant melanoma ${ }^{6,7}$. Histologically, non-caseating granulomata composed of epithelioid histiocytes with multinucleated giant cells are seen, but these can be indistinguishable from granulomatous inflammation from other causes. A series by Michael et $a^{8}{ }^{8}$ highlighted the difficulties encountered in making a definitive diagnosis of malignant neoplasm that mimics or occurs, in association with granulomata. In our study, out of 110 cases of cervical lympadenopathy 55 patients (50\%) FNAC report was specific. Rest 55 patients (non-specific lymphadenitis) underwent lymph node biopsy of which 5 were tuberculosis and 1 was lymphoma. Rest 49 cases were reactive. So FNAC was conclusive in total $(55+49)=104$ patients $(94.5 \%)$. This is supported by Michael et $\mathrm{al}^{8}$ and Reyes et $\mathrm{a} \mathbf{l}^{9}$. A suspicious clinical history of TB (pyrexia, night sweats, recent travel to endemic areas, no previous BCG vaccination) coupled with positive aspirate, blood, sputum or urine tests for AFB and good response to anti-tuberculous therapy supports the diagnosis of TB. One disadvantage is the inherent delay in culture result, but it is anticipated that as polymerase chain reaction and other amplification techniques become more common, detection time for the organism will shorten, improving the value of FNAC in clinical practice ${ }^{9}$.Although there is no single gold standard test, the important role of FNAC in histological diagnosis and its underutilisation was highlighted by Steel et $a^{10}$. We agree with the authors that FNAC used in conjunction with clinical findings, radiological and laboratory investigations can be a cost effective method. 


\section{Conclusion}

A significant number of cases of FNAC diagnosed granulomatous lymphadenitis have an identifiable underlying causal pathology. Our experience suggests that FNAC combined with clinical correlation is useful as a first line investigation. The high specificity of the technique helps to guide further investigation. It is also highly cost effective in the diagnosis of relapse in patients with malignancies.

\section{References}

1. Denenberg SN, Weisenburger DD, Nathwani BN, Winberg CD, Rappaport H.Multicentric angiofollicular lymph node hyperplasia: a clinicopathologic study of 16 cases. Hum Pathol 1985; 16:162-172

2. McAdams HP,Rosado-de- 1.A.G.Gonshal. Diagnosis of Tuberculosis. Journal of Indian Medical Association, March 2000; 98 (3): 57-61

3. Bhattacharya S. FNAC diagnosis of Tuberculosis an eight year study at Banglore, Indian journal of medical science. Nov 1998; 52 (11): 4985012
4. Fitzpatrich M.L. Micobacterlal cervical lymphadenitis. a review article. J.La state Med. soc. (USA), Nov 1996: 148(11): 451-454.

5. H.Harold Friedman Problem oriented Medical diagnosis. Lymphadenopathy, Clinics in Laboratory medicine. (USA), Des 1999; 19 (4): 237-241.

6. Kirn S.S. Application of PCR from the fine needle aspirates for the diagnosis of cervical tuberculous lymphadenitis. Journal of korean medical sciences, April 1999; 11 (2): 127-132.

7. Lee Y. Cervical tuberculous lymphadenitis: CT findings. Journal of Computer Assisted tomography (USA), May 1994; 18 (3): 370-375

8. Michael W Stanley, Out comes of FNAC, Clinics in Laboratory medicine. (USA), Des 1999 Vol 19 (4), Pages 773.

9. Reyes C.V. Metastasis of Unknown origin, role of F.N.A.C. Diagnostic Cytopathology, May 1998; 319-322,

10. Steel BL. FNAC. in the diagnosis of Lymphadenopathy. Journal A ta.cytology, Jan 1995; 39: 76-81. 